\title{
Ultrasound-guided pericapsular nerve group (PENG) hip joint phenol neurolysis for palliative pain
}

\author{
Andrés Rocha Romero, MD (1) · Gabriel Carvajal Valdy, MD • Angel Juarez Lemus, MD
}

Received: 3 June 2019/Revised: 18 June 2019/Accepted: 18 June 2019/Published online: 12 July 2019

(C) Canadian Anesthesiologists' Society 2019

\section{To the Editor,}

Cancer pain requires multidisciplinary management, and in most cases sufficient pain relief is possible using systemic multimodal analgesia. Nonetheless, complex cases often require interventional pain management strategies. The pericapsular nerve group (PENG) block is a recently described ultrasound-guided regional anesthesia technique targeting sensitive nerves from the anterior articular capsule of the hip (articular obturator branches, accessory obturator nerve, and articular femoral nerve branches), thus limiting potential mixed nerve block and quadricep or adductor muscle weakness. ${ }^{1}$

We present an 82-yr-old female diagnosed with a pathological left hip fracture related to metastatic epidermoid carcinoma and associated deep vein thrombosis (DVT). The patient was using apixaban 2.5 $\mathrm{mg}$ po bid. She was referred to our pain service because of severe pain on her left hip that was $8 / 10$ on a numeric rating scale limiting all basic activities (she was not able to sit). The patient had an Eastern Oncology Group 4 performance status and a Palliative Prognostic Index ${ }^{2}$ estimated survival shorter than six weeks. Her current pain treatment included transdermal buprenorphine 52.5 $\mu \mathrm{g} \cdot \mathrm{hr}^{-1}, 0.2 \mathrm{mg}$ buprenorphine sublingual tablets as rescue doses, paracetamol $750 \mathrm{mg}$, and pregabalin 150 mg daily. Lactulose was added to her treatment because of constipation.

\footnotetext{
A. Rocha Romero, MD $(\varangle)$ A A. J. Lemus, MD

Pain Clinic, Instituto Nacional de Cancerología, Mexico, Mexico

e-mail: rocharomeroandres@gmail.com

G. Carvajal Valdy, MD

Interventional Pain Unit, Centro Nacional de Control del Dolor y Cuidados Paliativos, San José, Costa Rica
}

A neurolytic PENG block was offered and written consent was obtained. She stopped her apixaban three days before the procedure. No previous diagnostic block was performed. The patient was placed in the supine position and a curvilinear low-frequency $8-3 \mathrm{MHz}$ ultrasound probe (Sonosite, USA) was placed over the anterior inferior iliac spine and aligned with the pubic ramus allowing identification of the iliopubic eminence, femoral artery, illiopsoas muscle, and pectineus muscle. A $10-\mathrm{cm} 22 \mathrm{G}$ spinal needle was advanced in a lateral to medial direction to place the tip in the musculofascial plane between the psoas tendon anteriorly and the pubic ramus posteriorly as described by Giron et al. ${ }^{1}$ Following negative aspiration, $10 \mathrm{~mL}$ of $6 \%$ phenol was injected in $5-\mathrm{mL}$ aliquots while confirming adequate fluid spread (Figure). Complete pain relief was obtained 30 min later and no motor weakness was documented. Two weeks later the patient continued to have relief, and was able to tolerate sitting in a wheelchair, though had referred pain in the popliteal area related to her DVT for which a lumbar plexus block was offered. The patient died two weeks later.

For chronic pain management, chemical ablation of the articular branches of the hip joint using alcohol has been recently published by Kwun-Tung et al. ${ }^{3}$ This was a retrospective study of 20 cases using this technique in frail elderly patients considered non-operable. They showed favourable results in movement-related pain on postintervention days 1 and $5(P<0.001)$, and in the degree of maximally tolerable hip flexion. Fifty percent of patients in this study managed to sit within the first five days after the procedure, without procedural adverse events.

Although published evidence is scarce, several regional ablative techniques have been described for hip cancerrelated pain. These include a neurolytic fascia iliaca block, or femoral nerve neurolysis, which may compromise motor 


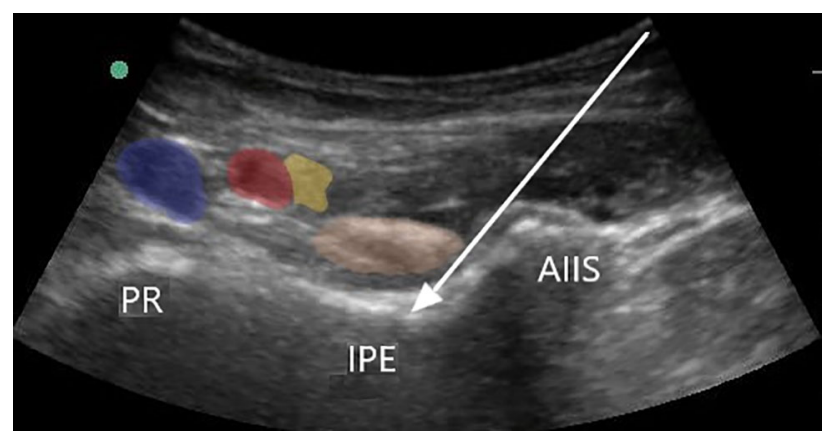

Figure Colourized sonographic capture of PENG neurolysis. The white arrow depicts needle trajectory. AIIS $=$ anteroinferior illiac spine; IPE = illiopubic eminence; $\mathrm{PENG}=$ pericapsular nerve group; $\mathrm{PR}=$ pubic ramus; yellow = femoral nerve; red = femoral artery; blue $=$ femoral vein; brown $=$ illiopsoas tendon

fibres and quadricep function. The presented case is, to our knowledge, the first case of a neurolytic PENG block using phenol being used to treat cancer-related pain. Contrasting with previous studies, we considered phenol a neurolytic agent. Although no specific studies have compared its properties with other conventional local anesthetics, phenol appears to have direct anesthetic properties, thus avoiding pain during neurolysis (or the need for previous local anesthetic infiltration) thus allowing less procedural related pain compared with alcohol neurolysis. ${ }^{4}$

This particular percutaneous technique may potentially be adapted to frail patients with short life expectancy where motor loss is not considered acceptable because of complications related to significant immobility. Nonetheless, it is important to recognize that this block has been associated with quadricep weakness in a small number of cases. ${ }^{5}$

The presented case illustrates the efficacy of a minimally invasive technique in a complex patient with cancer-related pain. Further studies are needed to confirm the motor sparing effect of the neurolytic PENG block and to clarify the optimal dose and concentration of phenol.

Conflicts of interest None declared.

Editorial responsibility This submission was handled by Dr. Hilary P. Grocott, Editor-in-Chief, Canadian Journal of Anesthesia.

\section{References}

1. Girón-Arango L, Peng PWH, Chin KJ, Brull R, Perlas A. Pericapsular nerve group (PENG) block for hip fracture. Reg Anesth Pain Med 2018; 43: 859-63.

2. Morita T, Tsunoda J, Inoue $S$, Chihara $S$. The palliative prognostic index: a scoring system for survival prediction of terminally ill cancer patients. Support Care Cancer 1999; 7: 128-33.

3. Kwun-Tung Ng T, Chan WS, Peng PW, Sham P, Sasaki S, Tsui HF. Chemical hip denervation for inoperable hip fracture. Anesth Analg 2019; DOI: https://doi.org/10.1213/ane.0000000000004172.

4. Guo S, Lam JW. Ablative techniques. In: Gulati A, Puttaniah V, Bruel BM, Rosenberg WS, Hung JC (Eds). Essentials of Interventional Cancer Pain Management, 1st ed. Springer Nature Switzerland; 2019: 141-3.

5. Yu HC, Moser JJ, Chu AY, Montgomery SH, Brown N, Endersby $R V$. Inadvertent quadriceps weakness following the pericapsular nerve group (PENG) block. Reg Anesth Pain Med 2019; 44I: 611-3.

Publisher's Note Springer Nature remains neutral with regard to jurisdictional claims in published maps and institutional affiliations. 